Musées, Patrimoine et Culture scientifiques et techniques

$183 \mid 2019$

mai-juin 2019

\title{
Exposer le monstrueux et interroger son rôle dans l'espace social contemporain
}

\author{
Catherine Gauthier
}

\section{OpenEdition}

\section{Journals}

Édition électronique

URL : http://journals.openedition.org/ocim/2443

DOI : $10.4000 /$ ocim. 2443

ISSN : 2108-646X

\section{Éditeur}

OCIM

Édition imprimée

Date de publication : 1 mai 2019

Pagination : 18-23

ISSN : 0994-1908

Référence électronique

Catherine Gauthier, «Exposer le monstrueux et interroger son rôle dans l'espace social

contemporain », La Lettre de I'OCIM [En ligne], 183 | 2019, mis en ligne le 01 juin 2020, consulté le 22 mars 2021. URL : http://journals.openedition.org/ocim/2443 ; DOI : https://doi.org/10.4000/ocim. 2443

Ce document a été généré automatiquement le 22 mars 2021.

Tous droits réservés 


\section{Exposer le monstrueux et interroger son rôle dans l'espace social contemporain}

Catherine Gauthier

L'affiche de l'exposition illustre le couple « monstre naturel/monstre artificiel » et par extension la coproduction La Casemate/Muséum de Grenoble.

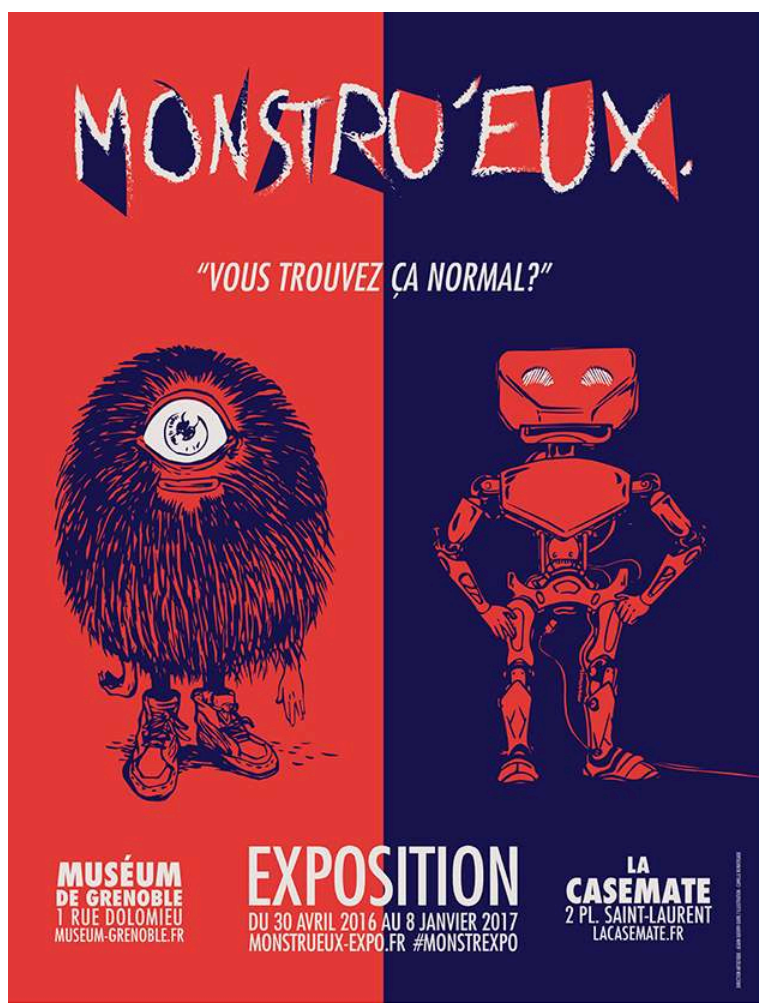

(c) ClickAndWatch/Muséum de Grenoble 


\section{Le musée, reflet d'une société contemporaine qui débat}

1 De nombreuses injonctions contradictoires ont poussé les musées à prudemment déserter le champ des controverses socio-scientifiques, au risque d'apparaître coupés des préoccupations de nos concitoyens: refléter les préoccupations de la société contemporaine tout en valorisant des collections issues de disciplines scientifiques éteintes ; produire un discours « expert » tout en séduisant le plus grand nombre, être le lieu du débat tout en évitant des polémiques que les financeurs du monde muséal, des collectivités publiques pour la plupart, craignent de ne pas pouvoir maîtriser....

2 Pourtant, exposer la question du réchauffement climatique ${ }^{1}$ ou de la «Sixième extinction » ne peut plus se faire sans se confronter à ces controverses qui ont envahi les espaces socio-numériques. À Grenoble, dans le cadre de l'élaboration du projet scientifique et culturel, la municipalité a souhaité que le muséum soit certes le lieu de l'explication de l'évolution du monde naturel contemporain à travers l'exemple alpin, mais aussi le lieu du débat. Comment refléter dès lors la pluralité des points de vue au cœur des expositions? Comme aborder certains sujets controversés tout en poursuivant notre mission de diffusion scientifique?

\section{Quelle légitimité pour celui qui parle?}

3 Au cœur des expositions, et en matière de sujet sensible plus qu'ailleurs, la question du «qui parle » est centrale 2 . Pour pouvoir interroger les questions les plus «sulfureuses » dont ils ont l'expertise, comme la survenue de malformations chez les nouveaux-nés, les institutions de diffusion scientifique doivent pouvoir s'appuyer sur un tissu d'acteurs en lien avec le sujet, à travers des partenariats construits parfois de longue date. C'est un préalable indispensable à l'exposition d'un discours «sensible»: le porter au côté du collectif des acteurs concernés.

4 Nous ne nous sentions pas légitimes en tant que naturalistes à parler seuls des monstres, ne serait-ce pour la question du monstre social sur laquelle nous achoppions; mais aussi parce la monstruosité convoque des réactions complexes, auxquelles nous ne pouvions échapper nous-mêmes et qui influenceraientt notre conception du projet si nous n'y prenions garde : "les réactions de celui qui le regarde [le monstre] sont alors diverses: d'abord l'inquiétude face à l'étrangeté puis la moquerie qui lui nie son humanité, la pitié... ou la reconnaissance de l'humain en lui ".

5 Exposer un sujet sensible, c'est donc identifier en amont toutes les dimensions de ce qui fera débat pour créer un collectif capable d'y répondre, légitimement ${ }^{4}$. Puis de porter, avec l'assentiment des acteurs, leur discours au cœur de l'exposition. 
Poppy le robot, figure totémique de la partie « monstre artificiel » (scénographie Ludovic Maggioni et Marguerite Pometko)

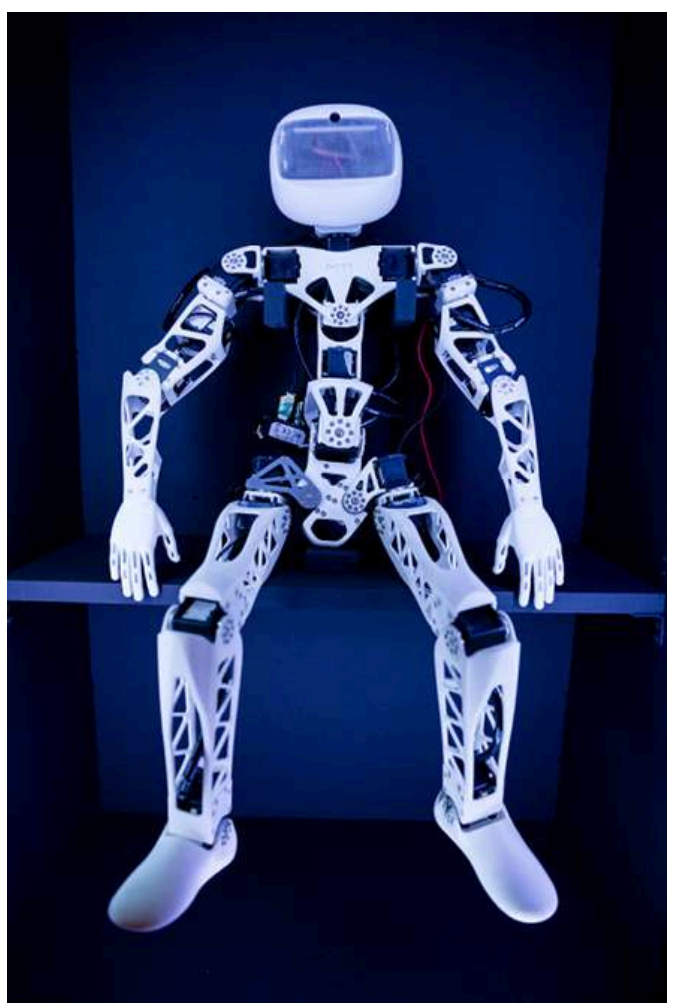

(C) Utopikphoto/La Casemate

\section{De la cartographie des controverses à la création d'un collectif de référents}

6 Tout d'abord, nous avons établi une cartographie des controverses pour identifier les arguments de chacun 5 . Ensuite, nous avons mobilisé sur plusieurs mois des spécialistes du domaine, "experts » assurant à l'institution de pouvoir offrir aux visiteurs un discours étayé issu de toutes les sphères de la société. Dans le cas de Monstrueux: vous trouvez ça normal ? il s'agissait de trouver des référents capables de nous expliquer ce qui fait norme dans la définition de l'humain aujourd'hui, que ce soit en biologie, en droit, en philosophie, dans le cinéma et les médias, dans la littérature jeunesse... Pour construire avec eux le contenu de l'exposition en lien avec les grands thèmes identifiés dans la carte des controverses.

7 Nous avons ensuite créé sous leur houlette une base documentaire de contenus de référence, dont un choix de collections. Nous avons par exemple collaboré avec un spécialiste des effets spéciaux du cinéma international sur la question de notre relation au monstre humain, et un enseignant agrégé de philosophie. Avec ce dernier, nous avons travaillé le discours sur le monstre «moral» pour pouvoir présenter dans l'exposition la classification des criminels de Cesare Lombroso ou traiter des auteurs de génocides. Sur celle de la transformation du génome humain, nous avons fait intervenir Pierre-Henri Gouyon dans un cycle de conférences académiques qui a servi de base à nos réflexions. 
Enfin, sur celle de la transformation de notre espèce à l'aune de l'émergence de l'intelligence artificielle et des robots, nous avons contacté le CCSTI La Casemate, référent national sur les questions des transformations sociales liées au numérique et drainant dans son sillage des organismes comme l'Inria (Institut national de recherche en informatique et en automatique). Laurent Chicoineau nous a alors proposé de coproduire l'exposition ${ }^{6}$ et de la présenter sur les deux sites (La Casemate et le muséum), soit sur plus de $400 \mathrm{~m}^{2}$, en traitant la question du monstre artificiel à La Casemate, respectant ainsi les attentes présupposées de nos publics? ${ }^{7}$.

\section{Constitution de l'équipe projet autour d'une priorité : montrer sans choquer}

9 Nous avons finalement identifié quatorze sujets à traiter, allant du monstre social au droit des robots en passant par la tératologie comme révélateur des grandes lois du vivant. Il nous fallait désormais mettre en scène cette diversité hétéroclite. Il nous est apparu important d'en conserver la richesse en définissant quatorze "scénettes " réparties en trois chapitres : le monstre imaginaire dont les faux monstres, le monstre naturel comme révélateur des lois et des normes et enfin le monstre artificiel créé par l'homme.

Restait à recruter un scénographe qui saurait nous accompagner pour présenter sans choquer, mais aussi sans cacher, la monstruosité. Nous avons porté notre choix sur Anaïs Renversade dont le travail délicat a permis de rendre hommage aux nombreux monstres de l'exposition comme la grande vitrine de tératologie dont les spécimens du musée de l'École vétérinaire de Lyon, présentés « comme des bijoux de luxe » sur fonds de dentelle dans un blanc épuré. Nous avons confié la scénographie de la partie des monstres imaginaires et des "faux» à Camille Renversade, auteur d'une série d'ouvrages sur les aventures du club des chasseurs de l'étrange, club de dandys cryptozoologues parcourant le monde à la recherche des monstres les plus célèbres. Cette partie nous permettait d'engager le dialogue avec les visiteurs sur la question des « faux » monstres, comme ceux qui pullulent sur Internet, tels des calmars géants de plus de 50 mètres de long ou des enfants centenaires... Il l'a brillamment fait avec l'appui du service des collections du muséum, à travers un savant mélange de faux et de vrais spécimens. C'est paradoxalement cette partie de l'exposition qui nous a d'ailleurs valut certaines de nos plus virulentes critiques sur le livre d'or: quelques visiteurs étaient " choqués » de voir ainsi exposer des faux dans un musée.

11 Notre volonté de laisser aux artistes une place importante dans la conception du projet nous a permis d'illustrer certains sujets à travers leurs créations comme celles des étudiants du master de design de Villefontaine (Rhône), celles du collectif Coin, les Boxing dolls torturées de Laura Vicédo ou encore les mises en scène photographiques de Poppy le robot par Loïg, révélant notre irrépressible empathie pour les machines. 
L'emplacement dans le parcours de visite du fœtus de cyclope humain a été soigneusement étudié. C'est ailleurs le seul cadavre humain présenté en alcool dans l'exposition, les deux autres étant le crâne phrénologique du professeur Gall et le masque mortuaire du sérial killer Dumollard.

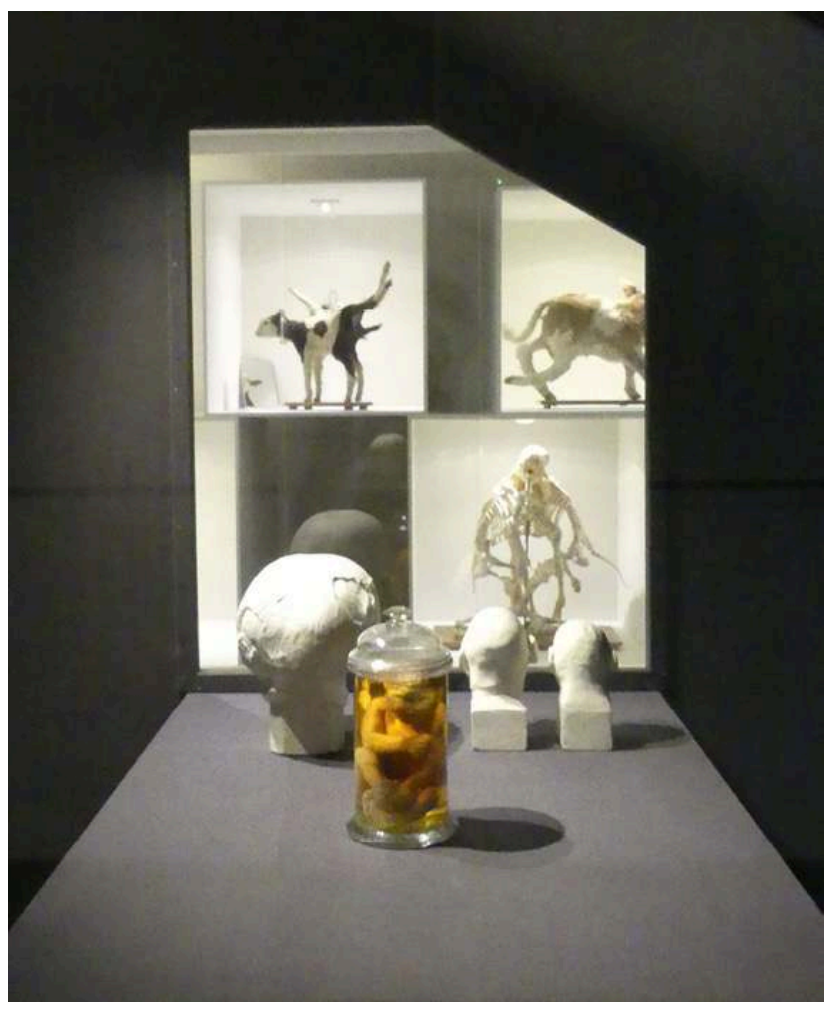

(c) Catherine Gauthier

\section{Mettre en scène des collections de monstres}

12 Afin de ne pas créer un "épuisement » émotionnel en exposant trop de cadavres de monstres tout au long du parcours, nous avons choisi de diversifier les spécimens exposés en alternant des taxidermies, des moulages en plâtre, des spécimens en alcool, des squelettes et des gravures. Ainsi, nous avons préféré exposer des moulages en plâtre de jeunes adultes microcéphales réalisés par le professeur Gall plutôt que des fœus en alcool, réservant aux visites guidées les images de bébés microcéphales nés au Brésil durant l'épidémie de virus Zika.

Pour les monstres les plus complexes, nous avons choisi des spécimens à forte valeur esthétique, comme un omphalosite de brebis au pelage immaculé et soyeux (appelé le " doudou ») ou de magnifiques squelettes enchevêtrés telles des sculptures, permettant d'approfondir la question des anomalies du plan de forme lors des visites.

Nous avons pensé spécifiquement l'emplacement des collections "sensibles» dans le parcours en isolant par exemple le foetus de cyclope en alcool sous un escalier dans une vitrine dédiée et en nous limitant dans toute l'exposition à ce seul spécimen pour ce qui concerne les cadavres de monstre humain. Pour les monstres de la collection de tératologie vétérinaire du professeur François-Xavier Lesbre, nous avons installé des miroirs pour que le visiteur puisse observer certains détails sans que la vue d'ensemble ne soit choquante, soulignant ainsi en seconde lecture certains caractères anormaux. 
Enfin, nous avons attaché une importance toute particulière à l'éclairage afin d'aboutir à une esthétisation apaisante indispensable à une observation pacifiée de la difformité.

Nous avons ainsi pu faire découvrir tout au long du parcours des collections exceptionnelles comme notre collection de poissons des abysses donnée par l'Ifremer (Institut français de recherche pour l'exploitation de la mer), les collections de criminologie du musée Testut Latarget d'anatomie et d'histoire médicale dont le masque mortuaire de Martin Dumollard, premier serial killer typologisé, des tirages des bustes raciaux du professeur Gall issus de nos collections d'anthropologie historique ou encore la collection de tératologie vétérinaire du professeur François-Xavier Lesbre datant de 1923.

Ptérodactyle capturé par le club des chasseurs de l'étrange : une création d'art taxidermie de Camille Renversade exposé dans son cabinet de cryptozoologie.

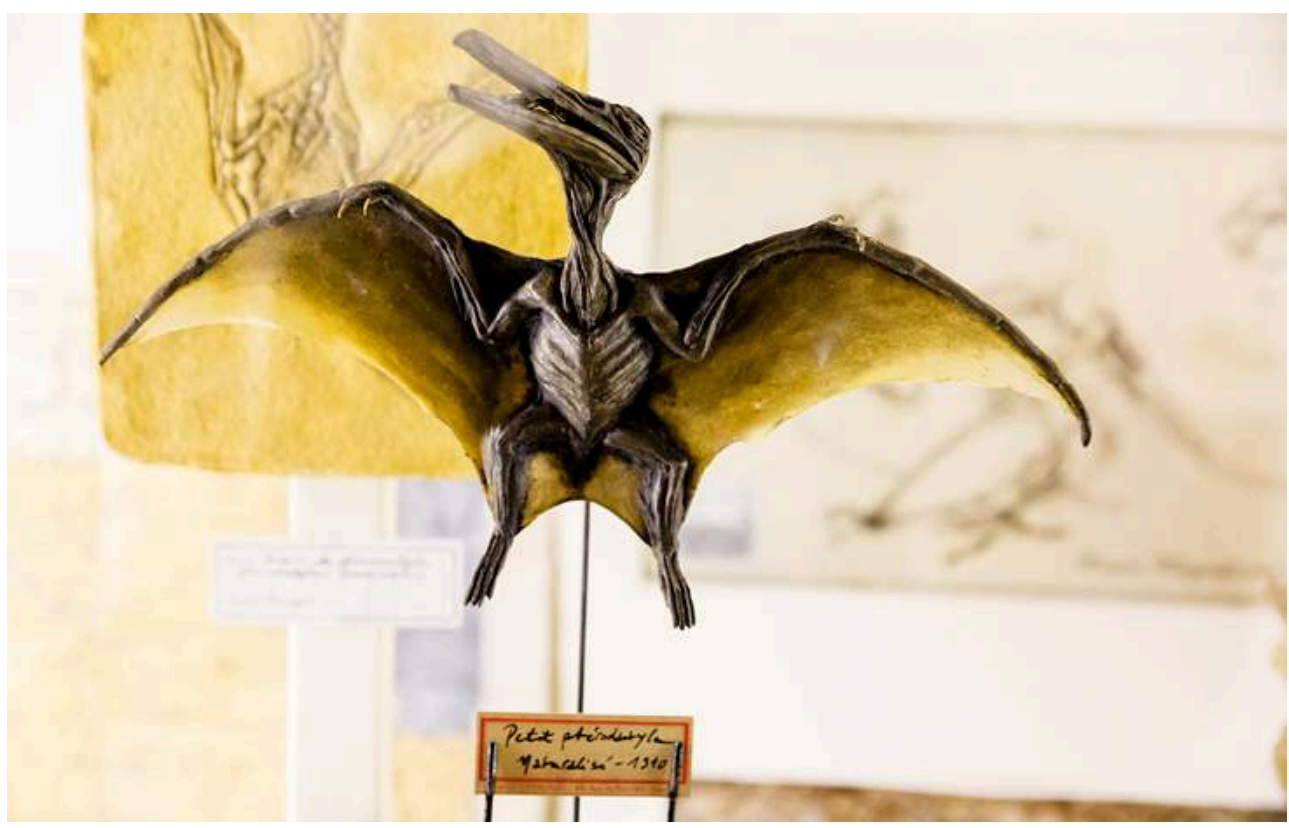

(c) Utopikphoto/La Casemate

\section{Le scriptovisuel de l'exposition}

De nombreuses images scientifiques, à l'instar de certaines collections, étaient particulièrement explicites. Nous avons donc décidé de présenter ces éléments sur des cubes d'enfants à tourner, laissant chacun découvrir à son rythme en tournant les cubes certains contenus. Alban Guerry-Suire, avec qui nous avions travaillé pour l'exposition Au fil des araignées, où montrer sans effrayer était déjà un enjeu, a élaboré en collaboration avec Anaïs et Camille Renversade une identité graphique originale : ils ont créé une famille de personnages monstrueux telle qu'il en existe dans la littérature pour enfants afin de permettre la dédramatisation du monstre humain. Le "petit frère ", un enfant cyclope souffrant d'hypertrichose, a été choisi pour transmettre tout au long du parcours des éléments clés du récit sous forme de bulles. 


\section{Et le débat dans tout ça?}

17 Nous avions anticipé via notre cartographie des controverses que l'exposition provoquerait certaines réactions, comme l'indignation face à l'exposition de cadavres humains... Les journalistes nous ont d'ailleurs interrogés sur la réaction de visiteurs présupposés trop sensibles pour pouvoir voir « en vrai » de tels monstres (remarque à laquelle il était facile de répondre au regard du grand succès des monstres dans la culture populaire et sur internet!).

Pour permettre aux visiteurs et plus largement aux personnes découvrant le sujet de s'exprimer, nous avons mis en place sur Echoscience une communauté numérique www.echosciences-grenoble.fr/communautes/monstrueux. Dans l'ensemble, les échanges y ont été très constructifs, comme durant les visites guidées de l'exposition et les conférences, les internautes nous adressant des articles, des liens vidéos ou des créations personnelles... Il nous est apparu au final que le soin que nous avions apporté au traitement général de la scénographie, mais aussi à la diversité des sujets traités nous avait finalement protégés du risque d'apparaître racoleurs ou provocateurs, une impossibilité lorsque l'on est une institution de diffusion scientifique reconnue.

Nous sommes d'ailleurs très fiers d'avoir poursuivi cette aventure avec de nombreux partenaires au-delà de l'exposition, que ce soit dans le cadre du plan de formation académique des enseignants où des séminaires sur la norme sont organisés depuis, ou dans celui de l'itinérance de l'exposition aux muséums de La Rochelle, Bourges, Blois et Auxerre.

Les textes et images de l'exposition ont été pour grande partie placés sur des « tournettes " que chacun pouvait manipuler à son rythme, pouvant ainsi s'attarder ou non sur certains contenus explicites. Ici, la tournette des robots à La Casemate.

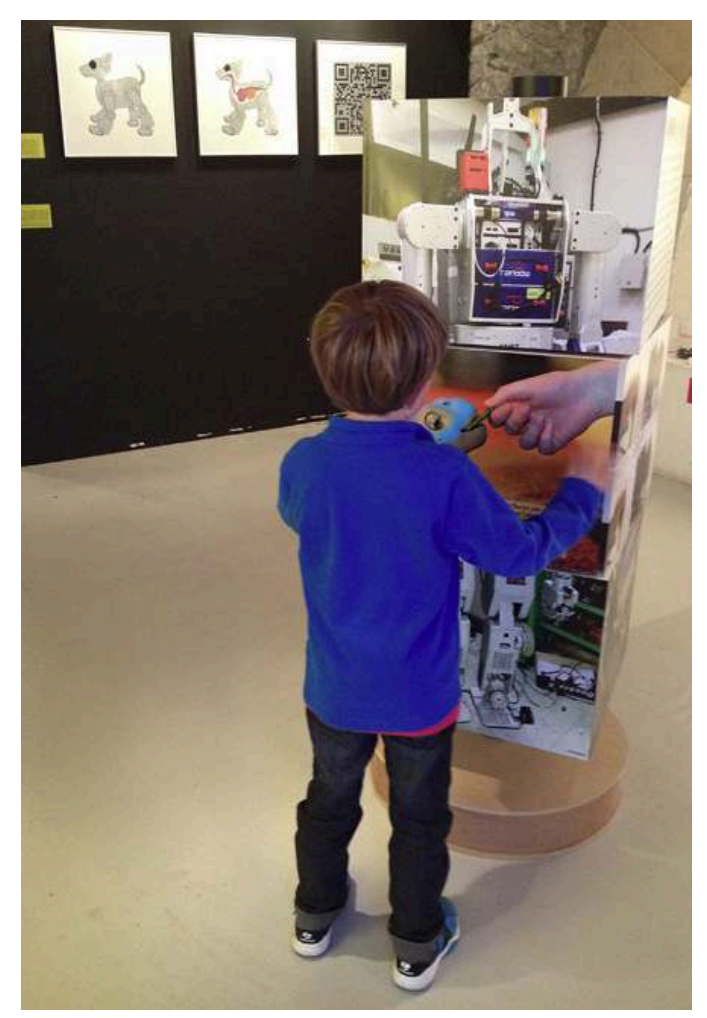

(c) Catherine Gauthier 
Le nid des webcams agitées et « pioupioutantes » du Collectif Coin.

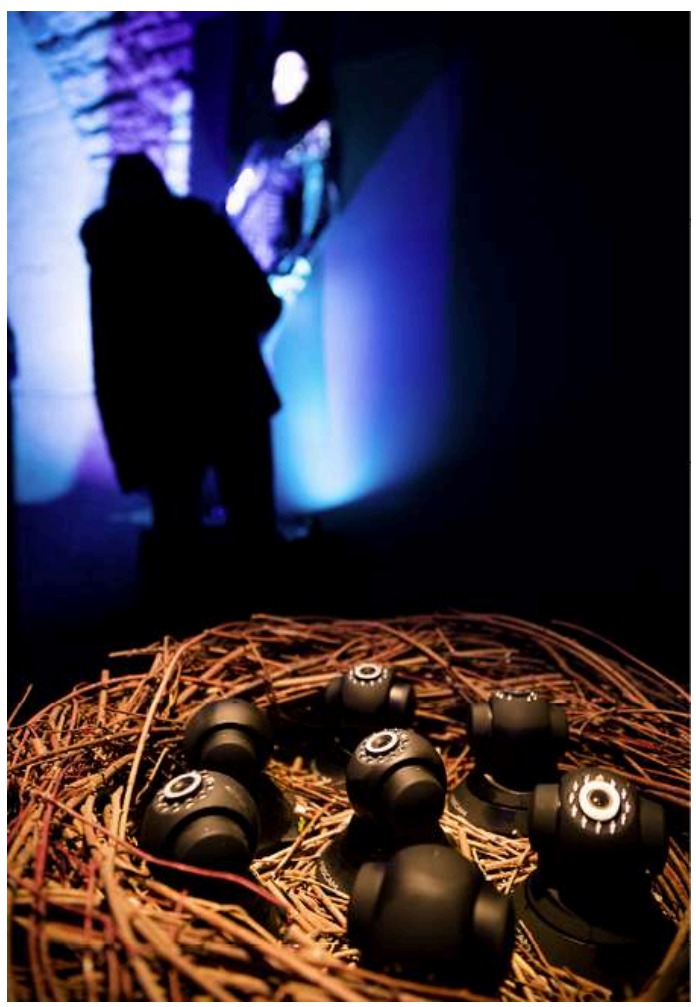

(C) La Casemate

\section{Conclusion}

Exposer un sujet sensible demande un engagement particulier de la part d'une institution, mais aussi de tous ses partenaires. Le commissaire de l'exposition est particulièrement exposé car il doit non seulement animer un collectif de référents issu d'horizons variés, mais aussi accueillir, de la part de son équipe, de ses tutelles, des usagers ou de la presse des avis parfois tranchés. S'exposer ainsi n'est pas toujours évident, et demande d'avoir en amont travaillé une grande diversité de sujets, en s'appuyant sur une cartographie des controverses et sur un collectif d'acteurs reconnus sur ces questions.

21 Mais c'est aussi à ce prix il me semble que les musées de science continueront d'être perçus comme des espaces de débat et de diffusion crédibles où l'on se rend pour voir "en vrai ", s'interroger, critiquer, s'étonner, et finalement pouvoir se forger une opinion sans s'en remettre uniquement aux publications les plus folles qui affluent sur Internet.

Et pour rassurer tous ceux qui hésitent à tenter cette aventure, il suffit de se dire qu'au fond, un sujet qui nous semble sulfureux aujourd'hui peut très vite devenir un sujet des plus consensuels.

L'auteur (commissaire de l'exposition) tient tout particulièrement à remercier pour leur participation à ce projet les équipes du muséum et de La Casemate (Ludovic Maggioni cocommissaire de l'exposition), Rémi Courrier, professeur agrégé de philosophie de l'Académie de 
Grenoble, le Club des chasseurs de l'étrange, le musée de La Résistance du département de l'Isère, le musée des Sciences Médicales du CHU de La Tronche, le musée de l'École Vétérinaire de Lyon, le musée Testut Latarget d'Histoire médicale, les Bibliothèques municipales de Grenoble, l'Institut de Communication et de Médiation de Grenoble.

\section{BIBLIOGRAPHIE}

Ancet, P. Phénoménologie des corps monstrueux. Éditions de la Réunion des musées nationaux et Gallimard, Paris, 1993.

Bensoussan, A. et Bensoussan, J. Droit des robots : orientations juridiques. Éditions Larcier, 2015.

Boaistuau, P. Histoires prodigieuses. Réédition de l'édition originale de 1560 avec ses illustrations, préface inédite d'Y. Florenne et glossaire des mots tombés en désuétude. Club Français du Livre, 1961.

Canguilhem, G. La monstruosité et le monstrueux, in La connaissance de la vie. Vrin, 1998.

Cryptozoologie : la science des animaux qui n'existent (peut-être) pas, Revue Espèces, $\mathrm{n}^{\circ} 17$.

Duhamel, B. L'œuvre tératologique d'Étienne Geoffroy Saint-Hilaire, Revue d'histoire des sciences, 1972, tome $25 \mathrm{n}^{\circ} 4$, pp. 337-346.

Lombroso, C. L'homme criminel. Étude anthropologique et psychiatrique. Traduit par Régnier et Bournet, Paris : Alcan Éditeur, 1887.

Martin, E. Histoire des monstres depuis l'Antiquité jusqu'à nos jours. Paris : C. Reinwald et Cie Éditeur 1880, p. 224. Réédition Jérôme Million Éditeur, Grenoble, 2002.

Montaigne, M. (de) Des boiteux. Essais III, 11. Texte modernisé, d'après l'exemplaire de Bordeaux : http://francaisdictionnairesimple.unblog.fr/2016/08/19/des-boiteux-montaigne/.

Péju, P. Le Monstrueux. Gallimard Jeunesse, 2007.

Wolff, É. Recherches sur la structure d'omphalocéphales obtenus expérimentalement, Archives d'anatomie, d'histologie et d'embryologie, tome 8, 1934, pp. 229-262.

\section{NOTES}

1. Davallon, J., Grandmont, G. et Schiele, B. L'environnement entre au musée. Musée de la civilisation/Centre Jacques Cartier/Édition des Presses Universitaires de Lyon, 1992.

2. Poli, M.-S. Le texte au musée : une approche sémiotique. L'Harmattan, 2002.

3. Nouailles, B. Le monstre ou le sens de l'écart. Essai de philosophie de la vie à partir des leçons de tératologie de G. et I. Saint-Hilaire. Université Blaise Pascal, Clermont-Ferrand II, 2012.

4. Molinatti, G. et Girault, Y. Comment les musées et centres de sciences s'exposent aux controverses socio-scientifiques. CNRS Éditions, $n^{\circ} 61,2013 / 3$.

5. La cartographie des controverses permet d'identifier les arguments des uns et des autres, l'adaptation des sociétés aux changements sociaux, la question de l'influence de ces questions sur 
les communautés de chercheurs/producteurs de connaissance (lobbying, censure...), voir Latour, B. et Venturini, T. La cartographie des controverses. Sciences-Po Paris : Medialab, 2008.

6. Nous avions déjà coproduit avec La Casemate une exposition d'art science en 2014 appelée Thinkrotron : la chambre d'écho.

7. Dans sa thèse de doctorat sur l'exposition itinérante La Différence et ses publics, Nathalie Candito démontre que l'horizon d'attente du visiteur quant à ce qu'il va découvrir en visitant une exposition diffère selon le lieu où elle est présentée.

\title{
RÉSUMÉS
}

Cette contribution interroge la capacité des musées à pouvoir présenter des controverses socioscientifiques dans des expositions de discours, en écho aux préoccupations des sociétés contemporaines. Elle témoigne plus précisément de la construction d'un discours muséal autour d'un sujet sensible, le monstre humain et la transformation de notre espèce, et de sa traduction muséographique.

\section{INDEX}

Mots-clés : Exposition, controverse, société

\author{
AUTEUR \\ CATHERINE GAUTHIER \\ Conservatrice du patrimoine scientifique, technique et naturel, professeure associée à \\ l'université Grenoble Alpes \\ catherine.gauthier@univ-grenoble-alpes.fr
}

